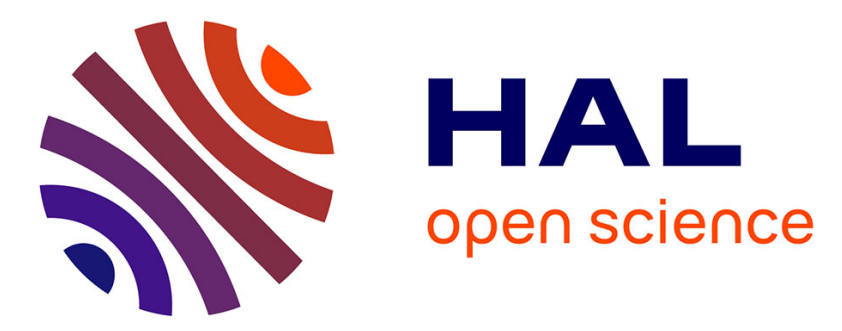

\title{
Nouveaux résultats sur l'origine des obsidiennes de Peiro Signado à Portiragnes (Hérault)
}

\author{
François Briois, Claire Manen, Bernard Gratuze
}

\section{To cite this version:}

François Briois, Claire Manen, Bernard Gratuze. Nouveaux résultats sur l'origine des obsidiennes de Peiro Signado à Portiragnes (Hérault). Bulletin de la Société préhistorique française, 2009, 106 (4), pp.809-811. 10.3406/bspf.2009.13899 . hal-01829791

\section{HAL Id: hal-01829791 \\ https://hal.science/hal-01829791}

Submitted on 5 Apr 2020

HAL is a multi-disciplinary open access archive for the deposit and dissemination of scientific research documents, whether they are published or not. The documents may come from teaching and research institutions in France or abroad, or from public or private research centers.
L'archive ouverte pluridisciplinaire HAL, est destinée au dépôt et à la diffusion de documents scientifiques de niveau recherche, publiés ou non, émanant des établissements d'enseignement et de recherche français ou étrangers, des laboratoires publics ou privés. 


\section{Nouveaux résultats sur l'origine des obsidiennes de Peiro Signado à Portiragnes (Hérault)}

Peiro Signado est un établissement néolithique ancien bien connu du Midi méditerranéen dont l'étude monographique est en cours. Nous communiquons ici les résultats des analyses effectuées sur les obsidiennes en incluant une réévaluation des analyses de provenance des premières pièces découvertes sur le site.

Localisé sur l'extrémité nord-occidentale d'un promontoire longiligne, en retrait du rivage actuel de la mer (environ $3 \mathrm{~km}$ ), le site de Peiro Signado dominait au début de l'Holocène un golfe marin aujourd'hui colmaté par des apports continentaux de la plaine alluviale de l'Orb (Ambert, 2007). Les premières fouilles effectuées en 1977 ont révélé un riche potentiel archéologique (Roudil et Grimal, 1978; Roudil et Soulier, 1983) et l'exploration du site a été reprise et achevée en 1996 et 1997 (Briois, 1997). Sur les $90 \mathrm{~m}^{2}$ de surface conservée du site, les restes d'un bâtiment ovalaire et plusieurs fosses adjacentes ont pu être mis en évidence (Briois et Manen, 2009). Ce gisement fait référence au Néolithique ancien à sillon d'impressions ou faciès Arene Candide qui a déjà été largement décrit depuis sa découverte (Roudil et Grimal, 1978; Roudil et Soulier, 1983; Manen, 2000 et 2002). L'industrie en silex est à base de lamelles et elle est caractérisée en particulier par des trapèzes à double troncature directe. La présence d'obsidienne est un phénomène suffisamment rare dans les contextes du Néolithique ancien du Midi de la France pour en souligner ici l'intérêt. Retrouvé sous la forme de lamelles, d'éclats et d'esquilles, ce matériau a manifestement été transformé à partir de petits volumes de matière première selon des principes comparables à celui qui a été appliqué au silex.

Le faciès lithique de Peiro Signado est comparable à celui du site voisin de Pont de Roque-Haute (Guilaine et al. dir., 2007). Les deux sites attestent de pratiques techniques en lien direct avec la sphère plus large du Néolithique ancien à céramique imprimée de l'aire italique, dont certains traits techniques conservent des caractères hérités du Castelnovien (Briois, 2005 et 2007). Daté entre 5700 et 5600 av. J.-C. (dates calibrées à 1 sigma), Peiro Signado fait partie des plus anciennes implantations néolithiques du Sud de la France (Briois et Manen, 2009).

\section{Le matériel étudié}

La série des obsidiennes de Peiro Signado est composée d'un total de 16 pièces, dont 3 proviennent des premières fouilles de 1977 ( 1 éclat et deux fragments de lamelles) et 13 des fouilles conduites en 1996 et 1997 (4 lamelles et fragments de lamelles, 3 éclats, 2 débris d'éclats, 4 esquilles). Douze pièces correspondent à une obsidienne relativement sombre et plutôt opaque et deux sont plus vitreuses et finement litées. Deux des lamelles découvertes par J.-L. Roudil avaient déjà fait l'objet d'analyses, l'une au laboratoire de Bradford et l'autre à l'université de Calabre, dont les résultats ont été publiés (Roudil et Soulier, 1983; Crisci et al., 1994).

Toutes les nouvelles analyses ont été réalisées au Centre Ernest-Babelon de l'Institut de recherche sur les archéomatériaux (CNRS/université d'Orléans). Elles ont été effectuées par spectrométrie de masse couplée à un plasma inductif avec prélèvement par ablation laser (LA-ICP-MS). Certaines d'entre elles ont également été faites par spectrométrie de fluorescence X (Gratuze, 1999; Biagi et al., 2007; Astruc et al., 2007) dans le cadre de la mise au point d'un protocole d'analyse par cette méthode.

\section{Résultats}

Les résultats obtenus (fig. 1) montrent que quatorze des seize obsidiennes étudiées ont une composition qui s'apparente à l'obsidienne de l'île de Palmarola. On retrouve dans ce groupe toutes les obsidiennes sombres et plutôt opaques. Il est important de noter ici que les deux lamelles d'obsidienne qui proviennent des fouilles de J.-L. Roudil appartiennent à ce groupe. Or, lors des études précédentes, ces obsidiennes avaient été identifiées comme liparotes (Roudil et Soulier, 1983; Crisci et al., 1994). Si on ne dispose pas des données provenant du laboratoire de Bradford, on notera cependant que

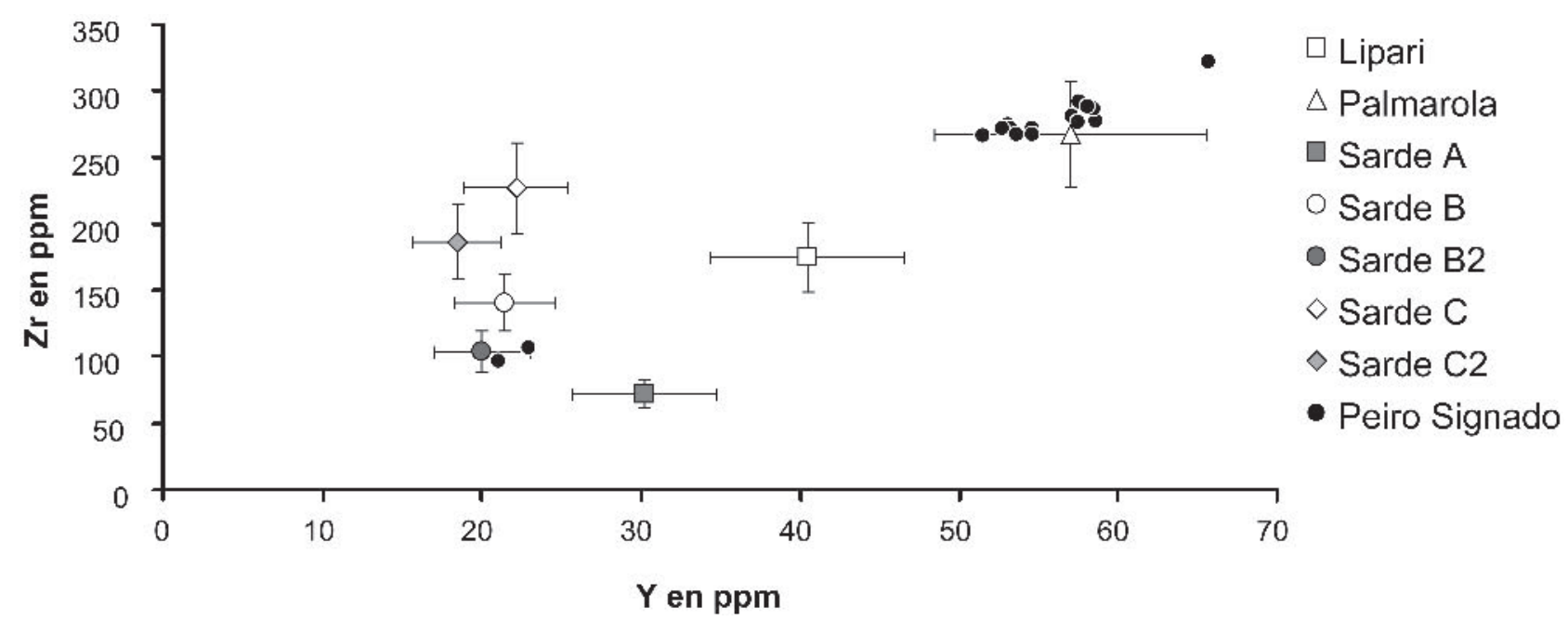

Fig. 1 - Représentation sur un diagramme binaire yttrium-zirconium des outils de Peiro Signado et des principales sources d'obsidienne de Méditerranée occidentale (teneurs mesurées par LA-ICP-MS). 


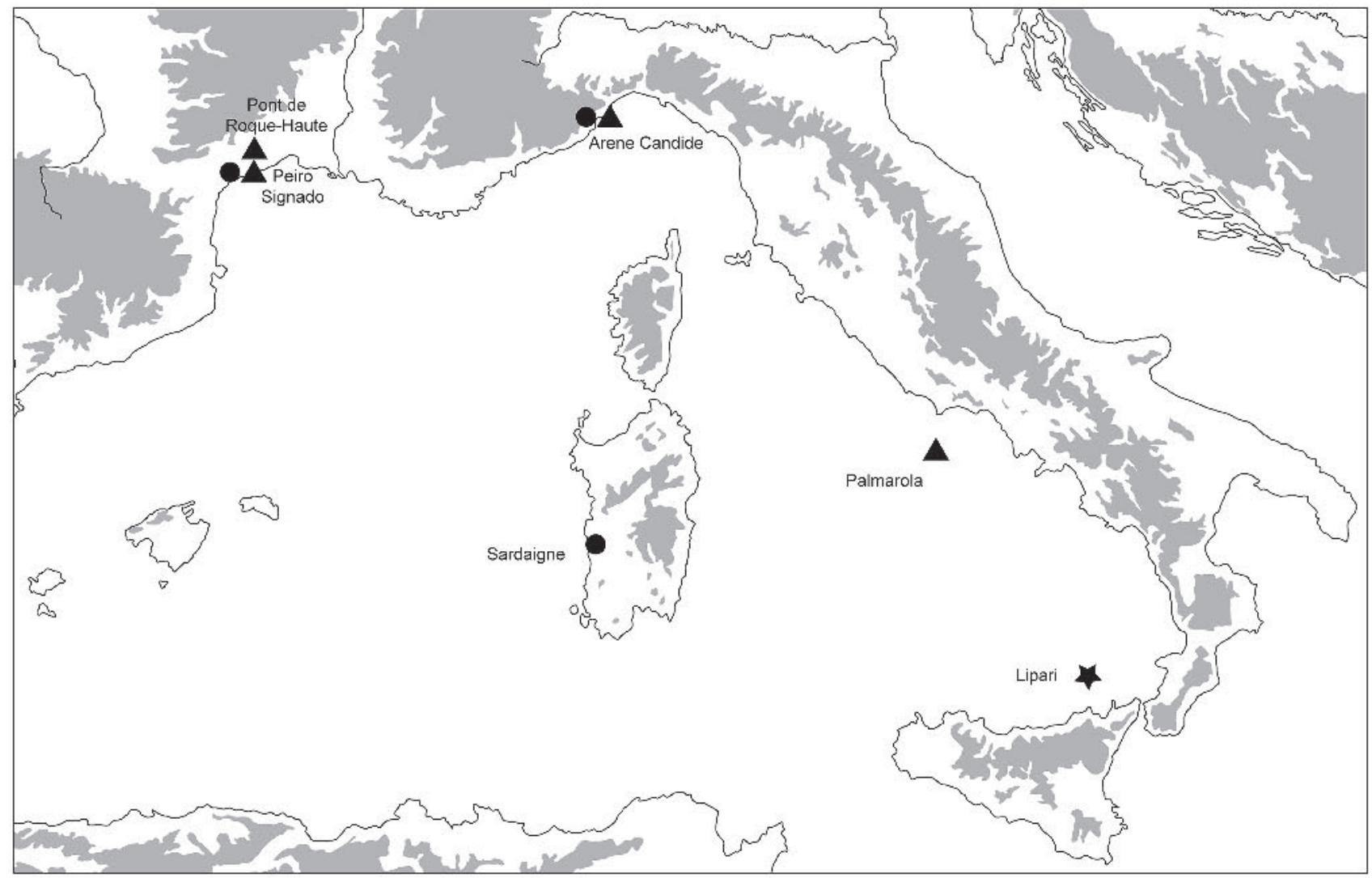

Fig. 2 - Localisation des sites de faciès impressa et des sources d'obsidienne de Méditerranée occidentale cités dans le texte (rond : Sardaigne; étoile : Lipari; triangle : Palmarola).

G.M. Crisci (université de Calabre) restait prudent sur les résultats de son analyse, il écrivait notamment ceci à son propos : «Deux échantillons, trouvés à Portiragnes et à Giribaldi, sortent légèrement du domaine de composition des obsidiennes de Lipari. S'ils viennent de cette source, ce qui est vraisemblable, cela signifie que les obsidiennes liparotes ont une composition moins homogène que ne le laissait supposer notre échantillonnage géologique» (Crisci et al., 1994). On remarquera toutefois que lors de cette étude, l'échantillonnage de G.M. Crisci ne comportait aucune obsidienne de Palmarola, ce qui peut expliquer l'erreur d'interprétation des données analytiques. Les fortes teneurs en rubidium, caractéristiques des obsidiennes de Palmarola, trouvées lors de nos analyses pour ces deux échantillons, ne laissent aucun doute quant à leur origine. Il s'avère donc aujourd'hui que ces deux obsidiennes proviennent elles aussi de Palmarola et non de Lipari comme cela a pu être publié (Roudil et Soulier, 1983; Crisci et al., 1994; Guilaine et Vaquer, 1994).

Les deux autres obsidiennes, plus claires et finement litées, se rapprochent de la composition des obsidiennes sardes provenant du gisement référencé sous le nom de Sarde B2. Il s'agit en fait de plusieurs petits gisements situés au nord-ouest du Monte Arci et qui produisent une obsidienne opaque ou transparente. On remarquera cependant que même si les teneurs en titane, yttrium, zirconium, niobium, cérium et lanthane sont identiques à celles de la source Sarde B2, les concentrations en baryum et strontium de ces échantillons se rapprochent plus de celles de l'obsidienne de la source Sarde A. Si l'origine sarde de ces deux échantillons ne fait aucun doute, leur composition apparaît donc cependant un peu marginale par rapport à nos échantillons de référence.

\section{Conclusions}

Les résultats obtenus sur la série de Peiro Signado mettent donc en évidence une double origine des obsidiennes (fig. 2) : la majorité des pièces provient de Palmarola; deux pièces seulement sont originaires de Sardaigne et plus probablement de l'ensemble de sources référencées sous le nom de sarde B2. Contrairement à ce qui avait été publié précédemment, ces analyses montrent l'absence effective de l'obsidienne liparote dans cet assemblage. La faible quantité d'obsidienne importée ne permet pas d'envisager l'existence d'un trafic qui s'inscrit dans la durée, pouvant impliquer, comme suggéré par J. Vaquer (2007), des relations régulières avec la région d'origine.

La détermination des origines de provenance concorde avec celle des couches du Néolithique ancien de la grotte des Arene Candide, où les obsidiennes de Sardaigne et celles de Palmarola sont associées à un effectif de pièces d'origine sarde légèrement plus important (Ammerman et Polglase, 1997). Sur ce site ligure, les trois sources sardes impliquées (SA, SB et SC) n'incluent toutefois pas la source B2 identifiée à Peiro Signado. Sur le site voisin de Pont de Roque-Haute, à Portiragnes, seule la source de Palmarola est attestée, mais il faut remarquer 
que cela tient peut-être à la faiblesse de l'effectif (Briois, 2007; De Francesco et Crisci, 2007). D'une manière générale, l'association entre l'obsidienne sarde et celle de Palmarola s'observe dans la partie nord de la côte tyrrhénienne et en Ligurie.

Ces résultats confirment que l'obsidienne de Palmarola a joué un rôle prépondérant en Méditerranée occidentale dès le premier Néolithique de faciès impressa, depuis l'aire tyrrhénienne jusqu'en Languedoc. L'implication des sources sardes, déterminée aux Arene Candide et confirmée par un petit nombre de pièces à Peiro Signado, est révélatrice du dynamisme et de la variété des réseaux de circulation de l'obsidienne dès la première moitié du $\mathrm{VI}^{\mathrm{e}}$ millénaire.

\section{RÉFÉRENCES BIBLIOGRAPHIQUES}

AMBERT P. (2007) - Les composantes géomorphologiques de Pont de Roque-Haute dans son contexte littoral, in J. Guilaine, C. Manen et J.-D. Vigne dir., Pont de Roque-Haute. Nouveaux regards sur la néolithisation de la France méditerranéenne, Archives d'écologie préhistorique, Toulouse, p. 53-68.

AMMERMAN A.J., POLGLASE C.R. (1997) - Analyses and descriptions of the obsidian collections from Arene Candide, in R. Maggi dir., Arene candide a functional and environnemental assessment of the Holocène sequence, Memorie dell'Istituto Italiano di Palethnologia Umana, 5, Il Calamo, Rome, p. 573-592.

ASTRUC L., GRATUZE B., PELEGRIN J., AKKERMANS P. (2007) From production to use: a parcel of obsidian bladelets at Sabi Abyad II, in L. Astruc, D. Binder et F. Briois dir., La diversité des systèmes techniques des communautés du Néolithique pré-céramique vers la caractérisation des comportements sociaux, Actes du $5^{e}$ colloque international sur les industries lithiques du Néolithique pré-céramique, éd. APDCA, Antibes, p. 327-341.

BIAGI P., GRATUZE B., BOUCETTA S. (2007) - New data on the archaeological obsidians from Banat and Transylvania (Romania). A short walk through the Balkans: The first farmers of the Carpathian basin and adjacent regions, Quaderno, 12, Societa per la Preistoria e Protoistoria della regione Friuli-Venezia Giulia, p. 129-139.

BRIOIS F. (1997) - Portiragnes, Peiro-Signado, Bilan scientifique de la région Languedoc-Roussillon, DRAC-SRA, p. 102-104.

BRIOIS F. (2005) - Les industries de pierre taillée néolithiques en Languedoc occidental. Nature et évolution des outillages entre les $6^{e}$ et $3^{e}$ millénaires av. J.-C., Monographies d'Archéologie méditerranéenne, $\mathrm{n}^{\circ} 20,341 \mathrm{p}$.

BRIOIS F. (2007) - L'industrie de pierre taillée, in J. Guilaine, C. Manen et J.-D. Vigne dir., Pont de Roque-Haute. Nouveaux regards sur la néolithisation de la France méditerranéenne, Archives d'écologie préhistorique, Toulouse, p. 77-81.

BRIOIS F., MANEN C. (2009) - L'habitat néolithique ancien de Peiro Signado à Portiragnes (Hérault), in A. Beeching et I. Sénépart dir., De la maison au village, L'habitat néolithique dans le Sud de la France et le Nord-Ouest méditerranéen, Actes de la table ronde des 23 et 24 mai 2003, Marseille/Musée d'Histoire de la ville de Marseille, Mémoire XLVIII, Société préhistorique française, p. 31-37.
CRISCI G.M., RICQ-DE BOUARD M., LANZAFRAME U., DE FRANCESCO A.-M. (1994) - Les obsidiennes du Midi de la France : nouvelle méthode d'analyse et provenance de l'ensemble des obsidiennes néolithiques du Midi de la France, Gallia Préhistoire, t. 36, p. 299-308.

DE FRANCESCO A.M., CRISCI G.M. (2007) - Provenance de l'obsidienne, in J. Guilaine, C. Manen et J.-D. Vigne dir., Pont de RoqueHaute. Nouveaux regards sur la néolithisation de la France méditerranéenne, Archives d'écologie préhistorique, Toulouse, p. 83-85.

GRATUZE B. (1999) - Obsidian characterisation by laser ablation ICPMS and its application to the prehistoric trade in the Mediterranean and the Near East: The sources and distribution of obsidian within the Aegean and Anatolia, Journal of Archaeological Sciences, vol. 26, p. $869-881$.

GUILAINE J., MANEN C., VIGNE J.-D. dir. (2007) - Pont de RoqueHaute (Portiragnes, Hérault). Nouveaux regards sur la néolithisation de la France méditerranéenne, Archives d'Écologie préhistorique, Toulouse, $336 \mathrm{p}$.

GUILAINE J., VAQUER J. (1994) - Les obsidiennes du Midi de la France : Les obsidiennes à l'ouest du Rhône, Gallia Préhistoire, t. 36, p. 323-327.

MANEN C. (2000) - Implantation de faciès d'origine italienne au Néolithique ancien : l'exemple des sites «liguriens» du Languedoc, in M. Leduc, N. Valdeyron et J. Vaquer dir., Sociétés et espaces, Rencontres méridionales de Préhistoire récente, Toulouse, 1998, Archives d'écologie préhistorique, Toulouse, p. 35-42.

MANEN C. (2002) - Structure et identité des styles céramiques du Néolithique ancien entre Rhône et Ebre, Gallia Préhistoire, t. 44, p. 121-166.

ROUDIL J.-L., GRIMAL J. (1978) - Découverte d'une nouvelle civilisation du Néolithique ancien en Languedoc, Bulletin de la Société préhistorique française, t. $75, \mathrm{n}^{\circ} 4$, p. 101-103.

ROUDIL J.-L., SOULIER M. (1983) - Le gisement néolithique ancien de Peiro Signado (Portiragnes, Hérault). Etude préliminaire, Congrès préhistorique de France, XXI session, Montauban-Cahors, sept. 1979 , vol. 2, Société préhistorique française, p. 258-279.

VAQUER J. (2007) - Le rôle de la zone nord tyrrhénienne dans la diffusion de l'obsidienne en Méditerranée nord-occidentale au Néolithique, in A. D’Anna, J. Césari, L. Ogel et J. Vaquer dir., Corse et Sardaigne préhistoriques, relations et échanges dans le contexte méditerranéen, Actes du $128^{e}$ congrès national du CTHS de Bastia, 2003, Documents préhistoriques, n² 22, éd. du CTHS, Paris, p. 99 119.

François BRIOIS et Claire MANEN Centre de recherche sur la Préet Protohistoire de la Méditerranée

UMR 5608 du CNRS-TRACES

39, allées Jules-Guesde, 31000 Toulouse

Bernard GRATUZE

Institut de recherche sur les Archéomatériaux UMR 5060 du CNRS

Centre Ernest Babelon 3 D, rue de la Férollerie, 45071 Orléans 